\title{
STATUTS POUR LA SECTION ASTIN DU COMITÉ PERMANENT
}

I. La section d'études actuarielles dans l'assurance de Risques non viagers (ASTIN) créée en conformité avec les Status du Comité Permanent, a comme objet la promotion de la recherche actuarielle dans l'assurance des Risques Divers.

La section ASTIN établira le contact entre actuaires, groupes d'actuaires et autres personnes de qualification requise, intéressées à ce domaine.

2. Il y aura trois classes de membres, soit:

Les membres ordinaires, qui étant membres du Comité Permanent, expriment le désir de se joindre à la section et sont enregistrés en conséquence;

Les membres spéciaux, c'est-à-dire les personnes de qualification requise qui peuvent être élues membres de la section sur la recommendation d'au moins deux membres ordinaires;

Les membres corporatifs, c'est-à-dire les institutions de tout genre requis qui s'intéressent aux affaires de ASTIN et contribuent à ses dépenses, à condition d'être élus membres de la section sur la recommandation d'au moins deux membres ordinaires.

Un membre peut démissionner n'importe quand, en prévenant dûment par écrit.

3. Les affaires de la section seront administrées par un comité de neuf membres maximum. Un ou deux de ces membres seront nommés par le Comité Permanent. Les autres membres seront élus par et parmi les membres ordinaires, tout les quatre ans. Le comité nommera un président, un secrétaire, un trésorier et un rédacteur dans son sein.

4. La section publiera de temps à autre, des documents sur des sujets en relation avec son objet et publiera également un bulletin contenant des notes d'un intérèt général pour les membres.

5. La section se réunira en conférences selon les décisions de son Comité, de telles conférences devant normalement avoir lieu au moins une fois tous les quatre ans. Les conférences seront ouvertes aux membres ordinaires et spéciaux de la section. Si la conférence a lieu concurremment avec un Congrès International d'Actuaires, elle sera ouverte à tous les membres de ce congrès. Le Comité peut inviter d'autres personnes de qualification requise à participer à de telles conférences.

6. Les cotisations dues par les membres seront fixées au cours des conférences à la simple majorité des membres ordinaires présents.

7. Tout changement de règlement et l'élection des membres du Comité sera déterminé par vote à la simple majorité des membres ordinaires présents à une réunion générale. Les recommandations en vue de modifier le règlement ou en vue de l'élection des membres du Comité seront envoyées au Secrétaire au moins trois mois avant la date du vote. Un changement de statuts ne deviendra effectif que lorsque le Comité Permanent aura donné son approbation. 